\title{
Four-step heating process for solid-phase crystallization of Ge leading to high carrier mobility
}

Takuto Mizoguchi, ${ }^{\text {a) }}$ Toshifumi Imajo, ${ }^{\text {a) }}$ Takashi Suemasu, and Kaoru Toko ${ }^{\text {b) }}$

Institute of Applied Physics, University of Tsukuba, 1-1-1 Tennodai, Tsukuba, Ibaraki 305-8573, Japan

Solid-phase crystallization is rapidly developing as a method for obtaining high-quality polycrystalline Ge layers. We propose a four-step heating process, focusing on postdeposition annealing (PDA), which is performed between the film deposition and crystallization. PDA at an appropriate temperature $\left(500{ }^{\circ} \mathrm{C}\right)$ reduces the grain size while improving the crystallinity of $\mathrm{Ge}$ (150 $\mathrm{nm}$ thickness), which leads to the reduction of grain boundary barrier height $(5 \mathrm{meV})$. The resulting hole mobility $\left(530 \mathrm{~cm}^{2} \mathrm{~V}^{-1} \mathrm{~s}^{-1}\right)$ is higher than that of any other semiconductor thin films $(<300 \mathrm{~nm})$ and even single-crystal Si wafers. These findings will further improve Ge thin-film transistors for advanced devices.

a) These authors contributed equally to this work.

b) Author to whom correspondence should be addressed.

Electronic mail: toko@bk.tsukuba.ac.jp 
Effective mobilities in Ge transistors have exceeded those in Si transistors. ${ }^{1-6}$ Although bulk Ge transistors have leakage currents due to their narrow band gaps, this problem has been solved by high-quality Ge-on-insulator (GOI) technology, such as mechanical transfer, ${ }^{7-10}$ oxidation-induced condensation, ${ }^{5,11}$ epitaxial growth on Si-on-insulator, ${ }^{12,13}$ lamp annealing, ${ }^{14,15}$ and rapid-melting growth. ${ }^{16-19}$ Low-temperature $\left(<600{ }^{\circ} \mathrm{C}\right)$ GOI technology has been studied vigorously for lowering the process costs and expanding the device application, including three-dimensional integrated circuits and flat-panel displays. Many growth techniques have been developed, such as laser annealing, ${ }^{20-23}$ chemical vapor deposition, ${ }^{24,25}$ and metal-induced crystallization. ${ }^{26-31}$ These methods have facilitated the preparation of polycrystalline Ge (poly-Ge) on insulators at low temperatures; however, the carrier mobility has remained below $200 \mathrm{~cm}^{2} \mathrm{~V}^{-1} \mathrm{~s}^{-1}$ because of the low crystallinity.

Solid-phase crystallization (SPC) is a simple method that consists of film deposition and heating. ${ }^{32-37}$ There has been considerable progress in the SPC of Ge in recent years, rendering an increased grain size of poly-Ge by heat deposition of amorphous Ge (a-Ge) precursors ${ }^{38}$ or addition of $\mathrm{Sn}$ in Ge. ${ }^{39-41}$ While poly-Ge is generally p-type due to the high hole concentration derived from defect-induced acceptors, ${ }^{44}$ n-type Ge has also been achived. ${ }^{42,43}$ The accumulation-mode p-channel transistors based on the p-type Ge exhibited field-effect mobility $\left(170 \mathrm{~cm}^{2} \mathrm{~V}^{-1} \mathrm{~s}^{-1}\right)$ higher than that of most of the p-channel Si transistors. ${ }^{45}$ Furthermore, we could increase the Hall mobility of holes to $620 \mathrm{~cm}^{2} \mathrm{~V}^{-1} \mathrm{~s}^{-1}$ by thickening Ge $(500 \mathrm{~nm})$, post annealing at $500{ }^{\circ} \mathrm{C}$, and inserting a $\mathrm{GeO}_{2}$ underlayer. ${ }^{46}$ However, a thinner film is preferred for suppressing the leakage current of the transistor. ${ }^{47}$

As mentioned above, we previously reported that the SPC of Ge has three heating processes to be controlled: film deposition, crystallization, and treatment post crystallization. These processes greatly influenced the crystallinity and electrical properties of the resulting poly-Ge. ${ }^{38,46}$ Here, we have added another heating process between film deposition and 
crystallization, that is, post-deposition annealing (PDA). PDA reduced the grain size of the 150 nm-thick poly-Ge layer while lowering the grain boundary barrier height, resulting in the highest hole mobility $\left(530 \mathrm{~cm}^{2} \mathrm{~V}^{-1} \mathrm{~s}^{-1}\right)$ among thin semiconductor films $(<300 \mathrm{~nm})$.

Figure 1(a) shows the schematic of the sample preparation. The a-Ge precursors $(150 \mathrm{~nm}-$ thick) were deposited on $\mathrm{SiO}_{2}$ glass substrates using the Knudsen cell of a molecular beam deposition system (base pressure: $5 \times 10^{-7} \mathrm{~Pa}$ ) while heating the samples at $125^{\circ} \mathrm{C}$ to densify Ge. ${ }^{38}$ After that, PDA was performed for $2 \mathrm{~h}$ in the same vacuum chamber. The PDA temperature, $T_{\mathrm{PDA}}$, ranged from $300{ }^{\circ} \mathrm{C}$ to $700{ }^{\circ} \mathrm{C}$. For comparison, we also prepared a sample without PDA $\left(T_{\mathrm{PDA}}=\mathrm{RT}\right)$. Next, the samples were loaded into a conventional tube furnace in a $\mathrm{N}_{2}(99.9 \%)$ atmosphere and annealed to induce SPC. The growth temperature (time), $T_{\mathrm{SPC}}$, ranged from $375^{\circ} \mathrm{C}(150 \mathrm{~h})$ to $450{ }^{\circ} \mathrm{C}(5 \mathrm{~h})$. Subsequently, we performed post-annealing (PA) at $500{ }^{\circ} \mathrm{C}$ for $5 \mathrm{~h}$ in an $\operatorname{Ar}(6 \mathrm{~N}$ purity) atmosphere.

The Raman spectra in Fig. 1(b) show sharp peaks near $300 \mathrm{~cm}^{-1}$ corresponding to crystalline Ge only for $T_{\mathrm{PDA}}=700{ }^{\circ} \mathrm{C}$, while no peaks are observed for $T_{\mathrm{PDA}}<700{ }^{\circ} \mathrm{C}$. Figure 1(c) shows that all samples with $T_{\mathrm{SPC}}=450{ }^{\circ} \mathrm{C}$ exhibit sharp peaks corresponding to crystal Ge. These results indicate that the nucleation of crystalline Ge start to form in the a-Ge layer above $600{ }^{\circ} \mathrm{C}$ in vacuum, while below $450{ }^{\circ} \mathrm{C}$ in $\mathrm{N}_{2}$ after exposing to air. This means that the crystallization energy of a-Ge is lower in $\mathrm{N}_{2}$ (after exposing to air) than in vacuum (before exposing to air). The same behavior was also observed in the SPC of Si, which explained that nitrogen atoms in the amorphous layer promote bond breaking and rearrangement. ${ }^{48}$ Therefore, PDA can apply a relatively high temperature to an a-Ge layer while maintaining the amorphous state. Raman shift and the full width at half maximum (FWHM) of the Ge-Ge peaks were evaluated from the Raman spectra and are summarized in Fig. 1(d). All peaks shift to lower wavenumbers than that of a bulk Ge substrate, originating from the tensile strain due to the difference in the thermal expansion coefficient between $\mathrm{Ge}$ and the $\mathrm{SiO}_{2}$ substrate. ${ }^{33,38}$ The peak 
shift increases till $T_{\mathrm{PDA}}$ reaches $700{ }^{\circ} \mathrm{C}$ and decreases thereafter. Conversely, FWHM decreases with increasing $T_{\mathrm{PDA}}$ and increases at $600{ }^{\circ} \mathrm{C}$. The sample with $T_{\mathrm{PDA}}=700{ }^{\circ} \mathrm{C}$, where nucleation occurred during PDA, shows a relatively small shift and large FWHM. These results indicate that the Ge crystals formed by PDA are of poor quality. Considering the sensitivity of Raman spectroscopy, the $T_{\mathrm{PDA}}=600{ }^{\circ} \mathrm{C}$ sample possibly contained some crystal nuclei and then exhibited a larger FWHM than the $T_{\mathrm{PDA}}=500{ }^{\circ} \mathrm{C}$ sample (Fig. $1(\mathrm{~d})$ ). Therefore, $T_{\mathrm{PDA}}=500{ }^{\circ} \mathrm{C}$ is almost optimal for high-quality SPC-Ge, which is the highest temperature that can maintain the amorphous state of Ge.

To investigate the effects of PDA on the SPC process, we evaluated the growth morphology using in-situ optical microscopy. The micrographs in Fig. 2(a) show the typical growth evolution of SPC. For both samples with $T_{\mathrm{PDA}}=500{ }^{\circ} \mathrm{C}$ and RT, we could observe the Ge nucleation that was followed by lateral growth of the domain with increasing annealing time. The Ge domain size saturated when the domains collided with each other. These micrographs clearly indicate that the crystallization is promoted by PDA. Figure 2(b) quantitatively shows the annealing-time dependence of the domain size, corroborating the micrographs. For the sample with $T_{\mathrm{PDA}}=500{ }^{\circ} \mathrm{C}$, the crystal nucleation started earlier than that for the $T_{\mathrm{PDA}}=\mathrm{RT}$ sample. Conversely, there was no significant change in the slopes corresponding to the lateral growth rate. These results indicate that PDA promotes nucleation rather than lateral growth and makes the eventual domain size small due to the high nuclear frequency.

The domains observed in the optical microscope are generally divided into several grains. ${ }^{38,43}$ Therefore, the actual grain size of the resulting poly-Ge was evaluated using electron backscattering diffraction (EBSD) analyses. The inverse pole figure (IPF) images in Fig. 3(a) show that the crystal orientation is almost random for all the samples, and the grain size depends on both $T_{\mathrm{PDA}}$ and $T_{\mathrm{SPC}}$. Figure $3(\mathrm{~b})$ quantitatively shows the grain size determined by the EBSD analyses. The samples with PDA had smaller grains than the samples without PDA $\left(T_{\mathrm{PDA}}=\mathrm{RT}\right)$, 
which is consistent with the results in Fig. 2 . For $T_{\mathrm{PDA}}<700^{\circ} \mathrm{C}$, a lower $T_{\mathrm{SPC}}$ rendered a larger grain size, which agrees with the basic behavior of SPC. ${ }^{33,38}$ Conversely, the sample with $T_{\mathrm{PDA}}$ $=700{ }^{\circ} \mathrm{C}$ had quite small grains regardless of $T$ sPC, which is likely due to the crystallization during the high-temperature PDA. The small grain size in the $T_{\mathrm{PDA}}=700{ }^{\circ} \mathrm{C}$ sample accounts for the large FWHM due to low crystallinity and the small peak shift due to the strain relaxation at the grain boundaries (Fig. 1) ${ }^{38}$ The sample with $T_{\mathrm{PDA}}=500{ }^{\circ} \mathrm{C}$ showed the smallest FWHM value (Fig. 1(d)), though its grain size was much smaller than that of the $T_{\mathrm{PDA}}=\mathrm{RT}$ sample. This behavior suggests that the $T_{\mathrm{PDA}}=500{ }^{\circ} \mathrm{C}$ sample has relatively few intragranular defects. Although the detailed mechanism is still unclear, PDA may allow the a-Ge layer to have a more crystalline atomic arrangement, which induces the subsequent SPC with less stress.

The electrical properties of the resulting poly-Ge layers were evaluated by the Hall effect measurements. All samples showed p-type conduction, similar to the conventional undoped SPC-Ge. ${ }^{33,38}$ As shown in Fig. 4(a), for all the samples, the hole concentration $p$ decreased after PA due to the passivation of the defect-induced acceptors. ${ }^{45}$ For both before and after PA, $p$ increases with $T$ PDA. Considering the $T$ PDA dependence of the grain size, this behavior is due to the increase of acceptors from the grain boundaries. Figure 4(b) shows that all the samples except $T_{\mathrm{PDA}}=700{ }^{\circ} \mathrm{C}$ exhibit hole mobility $\mu$ over $300 \mathrm{~cm}^{2} \mathrm{~V}^{-1} \mathrm{~s}^{-1}$. The limiting factors of $\mu$ are discussed as follows, taking the sample with $T_{\mathrm{PDA}}=\mathrm{RT}$ and $T_{\mathrm{SPC}}=375{ }^{\circ} \mathrm{C}$ before PA as an example. According to Matthiessen's rule, $\mu$ of the sample is expressed as

$$
\frac{1}{\mu}=\frac{1}{\mu_{\mathrm{P}}}+\frac{1}{\mu_{\mathrm{I}}}+\frac{1}{\mu_{\text {others }}}
$$

where $\mu \mathrm{P}, \mu \mathrm{I}$, and $\mu_{\mathrm{others}}$ are the hole mobilities limited by phonon scattering, impurity scattering, and the other scattering factors including grain boundary scattering, respectively. According to equation (1) and the relationship between $\mu$ and $p$ of single-crystal Ge, ${ }^{49} \mu \mathrm{P}, \mu \mathrm{I}$, and $\mu_{\text {others }}$ are 
determined to be 1900,1090 , and $760 \mathrm{~cm}^{2} \mathrm{~V}^{-1} \mathrm{~s}^{-1}$, respectively. These results indicate that the $\mu$ values of the current poly-Ge layers are limited by both grain boundary scattering and impurity scattering at RT. Figure 4(c) shows that $p$ decreases with decreasing $T$ due to the inactivation of the defect-induced acceptors. Conversely, $\mu$ increases with decreasing $T$ due to the reduction of the impurity scattering, and then begins to decrease due to the manifestation of grain boundary scattering. ${ }^{50}$ This behavior also indicates that $\mu$ is limited by both impurity scattering and grain boundary scattering near RT, while dominantly by grain boundary scattering at low temperatures. Figure 4(b) shows that, for each $T_{\mathrm{PDA}}$, the lower $T_{\mathrm{SPC}}$ provides a higher $\mu$ owing to the larger grain size, which implies a weaker grain boundary scattering. After PA, $\mu$ improves for all the samples owing to the decrease in the impurity scattering. The $T_{\mathrm{PDA}}=500{ }^{\circ} \mathrm{C}$ sample exhibits the highest $\mu$ value reaching $530 \mathrm{~cm}^{2} \mathrm{~V}^{-1} \mathrm{~s}^{-1}$. This is the highest value for Ge thin film $(<300 \mathrm{~nm})$ formed directly on the glass,${ }^{47}$ which is the advantage of fabricating a thin film transistor with a low leakage current.

We observed that PDA improves $\mu$ while reduces the grain size of Ge. To explain this behavior, we investigated the energy barrier height $\left(E_{\mathrm{B}}\right)$ of the grain boundary. According to the carrier conduction model in polycrystalline semiconductors proposed by Seto,${ }^{50}$ the carrier mobility limited by grain boundary scattering can be determined using the following equation:

$$
\mu=\frac{L q}{\sqrt{2 \pi m^{*} k T}} \exp \left(-\frac{E_{\mathrm{B}}}{k T}\right)
$$

where $T$ is the absolute temperature, $L$ is the grain size, $m^{*}$ is the effective mass, and $k$ is the Boltzmann constant. The trapped state density $\left(Q_{\mathrm{t}}\right)$ in the grain boundary can be determined using the following equation:

$$
Q_{\mathrm{t}}=\frac{\sqrt{8 \varepsilon N E_{\mathrm{B}}}}{q}
$$

where $N$ is the carrier concentration, $\varepsilon$ is the dielectric permittivity, and $q$ is the elementary charge. Figure 4(d) shows that for all samples, $\mu T^{1 / 2}$ moderately increases with decreasing $T$ and then begins to decrease, which is consistent with the $\mu$ behavior with respect to $T$ (Fig. 4(c)). 
Figure $4(\mathrm{e})$ shows that PDA reduces $E_{\mathrm{B}}$, whereas $Q_{\mathrm{t}}$ is almost the same. After PA, the $E_{\mathrm{B}}$ difference between the samples with and without PDA becomes more pronounced. According to equation (3), this behavior results from $E_{\mathrm{B}}$ reflecting $N$ and $Q_{\mathrm{t}}$, where PA lowers both $N$ and $Q_{\mathrm{t}}$. Because $\mu$ is limited by both impurity scattering and grain boundary scattering, the high $\mu$ of the $T_{\mathrm{PDA}}=500{ }^{\circ} \mathrm{C}$ sample is considered to originate from the proper balance between $N$ and $E_{\mathrm{B}}$

In conclusion, PDA was effective in increasing $\mu$ of thin $(150 \mathrm{~nm}) \mathrm{SPC}-\mathrm{Ge}$ on glass. PDA at an appropriate temperature $\left(500^{\circ} \mathrm{C}\right)$ reduced the grain size while improving crystallinity, which led to the reduction of $E_{\mathrm{B}}$ after PA. This resulted in a $\mu$ of $530 \mathrm{~cm}^{2} \mathrm{~V}^{-1} \mathrm{~s}^{-1}$, which is higher than any other Ge layers directly synthesized on glass without an underlayer and those with a film thickness below $300 \mathrm{~nm}$. Therefore, controlling the four heating processes is essential for modulating the crystallinity and electrical properties of the Ge layers. Ge thin films with higher $\mu$ than Si wafers will lead to the development of high-performance thin-film transistors for three-dimensional integrated circuits and flat-panel displays.

This work was financially supported by a Grant-in-aid for JSPS Research Fellow (No. 19J21034), the Yazaki Memorial Foundation for Science, the TEPCO Memorial Foundation, and the JST PRESTO (No. JPMJPR17R7). The authors are grateful to Prof. T. Sakurai (University of Tsukuba) for the Hall effect measurements and Ms. T. Tawara and Mr. K. Tanigawa (University of Tsukuba) for their help with experiments at the Nanotechnology Platform. EBSD measurements were conducted at the International Center for Young Scientists in NIMS. 


\section{REFERENCES}

1 A. Nayfeh, C. O. Chui, T. Yonehara, and K. C. Saraswat, IEEE Electron Device Lett. 26, 311 (2005).

2 D. P. Brunco, B. De Jaeger, G. Eneman, J. Mitard, G. Hellings, a. Satta, V. Terzieva, L. Souriau, F. E. Leys, G. Pourtois, M. Houssa, G. Winderickx, E. Vrancken, S. Sioncke, K. Opsomer, G. Nicholas, M. Caymax, a. Stesmans, J. Van Steenbergen, P. W. Mertens, M. Meuris, and M. M. Heyns, J. Electrochem. Soc. 155, H552 (2008).

3 R. Pillarisetty, Nature 479, 324 (2011).

$4 \quad$ K. Yamamoto, T. Sada, D. Wang, and H. Nakashima, Appl. Phys. Lett. 103, 122106 (2013).

5 S. Takagi, R. Zhang, J. Suh, S.-H. Kim, M. Yokoyama, K. Nishi, and M. Takenaka, Jpn. J. Appl. Phys. 54, 06FA01 (2015).

$6 \quad$ A. Toriumi and T. Nishimura, Jpn. J. Appl. Phys. 57, 010101 (2018).

7 G. Taraschi, A. J. Pitera, and E. A. Fitzgerald, Solid. State. Electron. 48, 1297 (2004).

8 Y. Moriyama, K. Ikeda, Y. Kamimuta, M. Oda, T. Irisawa, Y. Nakamura, A. Sakai, T. Tezuka, Solid. State. Electron. 83, 42 (2013).

9 W. Mizubayashi, S. Noda, Y. Ishikawa, T. Nishi, A. Kikuchi, H. Ota, P.-H. Su, Y. Li, S. Samukawa, and K. Endo, Appl. Phys. Express 10, 026501 (2017).

10 K. Yu, F. Yang, H. Cong, L. Zhou, Q. Liu, L. Zhang, B. Cheng, C. Xue, Y. Zuo, and C. Li, J. Alloys Compd. 750, 182 (2018).

11 T. Maeda, K. Ikeda, S. Nakaharai, T. Tezuka, N. Sugiyama, Y. Moriyama, and S. Takagi, Thin Solid Films 508, 346 (2006).

12 V. D. Cammilleri, V. Yam, F. Fossard, C. Renard, D. Bouchier, P. F. Fazzini, L. Ortolani, F. Houdellier, and M. Hÿtch, Appl. Phys. Lett. 93, 043110 (2008).

13 O. Nakatsuka, N. Tsutsui, Y. Shimura, S. Takeuchi, A. Sakai, and S. Zaima, Jpn. J. Appl. Phys. 49, 04DA10 (2010).

14 K. Usuda, Y. Kamata, Y. Kamimuta, T. Mori, M. Koike, and T. Tezuka, Appl. Phys. Express 7, 056501 (2014).

15 Y. Kamata, M. Koike, E. Kurosawa, M. Kurosawa, H. Ota, O. Nakatsuka, S. Zaima, and T. Tezuka, Appl. Phys. Express 7, 121302 (2014).

16 J. Feng, G. Thareja, M. Kobayashi, S. Chen, A. Poon, Y. Bai, P.B. Griffin, S.S. Wong, Y. Nishi, and J.D. 
Plummer, IEEE Electron Device Lett. 29, 805 (2008). Soc. 157, H371 (2010). Hamaya, J. Appl. Phys. 123, 215704 (2018). (2011). Phys. Express 11, 101305 (2018).

S. Hu, P. W. Leu, A. F. Marshall, and P. C. McIntyre, Nat. Nanotechnol. 4, 649 (2009).

K. Toko, Y. Ohta, T. Tanaka, T. Sadoh, and M. Miyao, Appl. Phys. Lett. 99, 32103 (2011).

T. Hosoi, Y. Suzuki, T. Shimura, and H. Watanabe, Appl. Phys. Lett. 105, 173502 (2014).

H. Watakabe, T. Sameshima, H. Kanno, and M. Miyao, Thin Solid Films 508, 315 (2006).

W. Yeh, H. Chen, H. Huang, C. Hsiao, and J. Jeng, Appl. Phys. Lett. 93, 094103 (2008).

K. Sakaike, S. Higashi, H. Murakami, and S. Miyazaki, Thin Solid Films 516, 3595 (2008).

H. A. Kasirajan, W.-H. Huang, M.-H. Kao, H.-H. Wang, J.-M. Shieh, F.-M. Pan, and C.-H. Shen, Appl.

T. Matsui, M. Kondo, K. Ogata, T. Ozawa, and M. Isomura, Appl. Phys. Lett. 89, 142115 (2006).

M. Tada, J.-H. Park, D. Kuzum, G. Thareja, J. R. Jain, Y. Nishi, and K. C. Saraswat, J. Electrochem.

Z. Wang, L. P. H. Jeurgens, W. Sigle, and E. J. Mittemeijer, Phys. Rev. Lett. 115, 016102 (2015).

S. Hu, A. F. Marshall, and P. C. McIntyre, Appl. Phys. Lett. 97, 082104 (2010).

J.-H. Park, K. Kasahara, K. Hamaya, M. Miyao, and T. Sadoh, Appl. Phys. Lett. 104, 252110 (2014).

T. Suzuki, B. M. Joseph, M. Fukai, M. Kamiko, and K. Kyuno, Appl. Phys. Express 10, 095502 (2017).

H. Higashi, K. Kudo, K. Yamamoto, S. Yamada, T. Kanashima, I. Tsunoda, H. Nakashima, and K.

K. Toko and T. Suemasu, J. Phys. D. Appl. Phys. 53, 373002 (2020).

T. Sadoh, H. Kamizuru, A. Kenjo, and M. Miyao, Appl. Phys. Lett. 89, 192114 (2006).

K. Toko, I. Nakao, T. Sadoh, T. Noguchi, and M. Miyao, Solid-State Electron. 53, 1159 (2009).

C.-Y. Tsao, J. Huang, X. Hao, P. Campbell, and M. A. Green, Sol. Energy Mater. Sol. Cells 95, 981

H.-W. Jung, W.-S. Jung, H.-Y. Yu, and J.-H. Park, J. Alloys Compd. 561, 231 (2013).

S. Kabuyanagi, T. Nishimura, K. Nagashio, and A. Toriumi, Thin Solid Films 557, 334 (2014).

A. Hara, Y. Nishimura, and H. Ohsawa, Jpn. J. Appl. Phys. Part 1 56, 03 BB01 (2017).

K. Toko, R. Yoshimine, K. Moto, and T. Suemasu, Sci. Rep. 7, 16981 (2017).

W. Takeuchi, N. Taoka, M. Kurosawa, M. Sakashita, O. Nakatsuka, and S. Zaima, Appl. Phys. Lett. 107, 22103 (2015). 
$41 \quad$ K. Moto, R. Yoshimine, T. Suemasu, and K. Toko, Sci. Rep. 8, 14832 (2018).

D. Takahara, K. Moto, T. Imajo, T. Suemasu, and K. Toko, Appl. Phys. Lett. 114, 082105 (2019).

43 M. Saito, K. Moto, T. Nishida, T. Suemasu, and K. Toko, Sci. Rep. 9, 16558 (2019).

44 H. Haesslein, R. Sielemann, and C. Zistl, Phys. Rev. Lett. 80, 2626 (1998).

45 K. Moto, K. Yamamoto, T. Imajo, T. Suemasu, H. Nakashima, and K. Toko, Appl. Phys. Lett. 114, 212107 (2019).

46 T. Imajo, K. Moto, R. Yoshimine, T. Suemasu, and K. Toko, Appl. Phys. Express 12, 015508 (2019).

47 C. Xu, X. Gong, M. Miyao, and T. Sadoh, Appl. Phys. Lett. 115, 042101 (2019).

48 N. Makino and Y. Shigeta, Thin Solid Films 658, 61 (2018).

49 M. Prince, Phys. Rev. 92, 681 (1953).

$50 \quad$ J.W.Y. Seto, J. Appl. Phys. 46, 5247 (1975). 
(a)
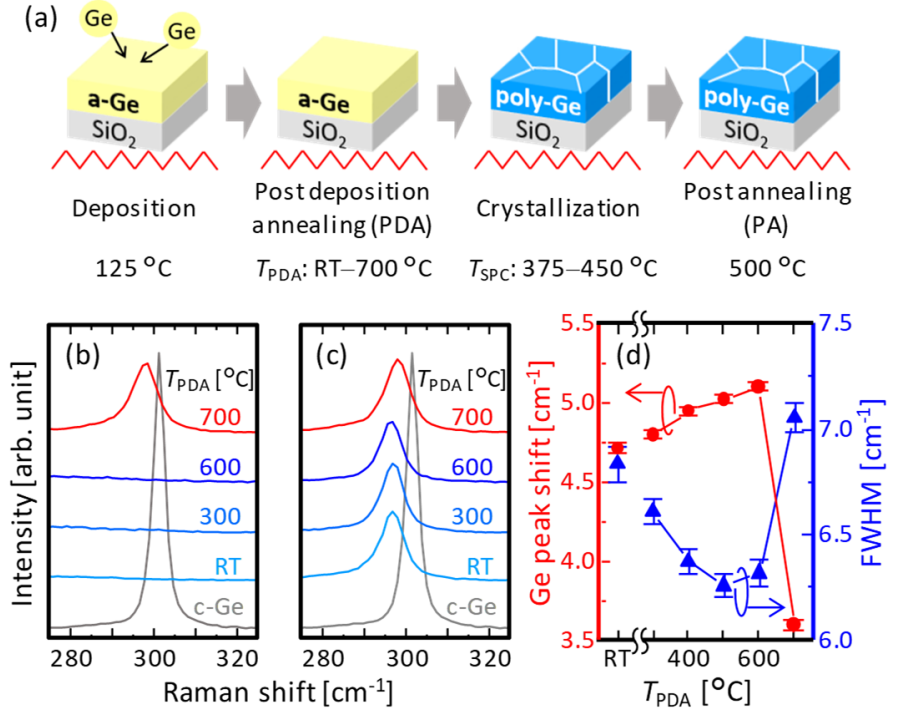

FIG. 1. (a) Schematic of sample preparation process. Raman spectra after (b) PDA and (c) SPC $\left(T_{\mathrm{SPC}}=450{ }^{\circ} \mathrm{C}\right)$ for various $T_{\mathrm{PDA}}$. The spectra of bulk-Ge is shown for comparison. (d) Ge peak shifts from that of bulk-Ge and FWHMs of the Ge peaks as a function of $T_{\mathrm{PDA}}$. 

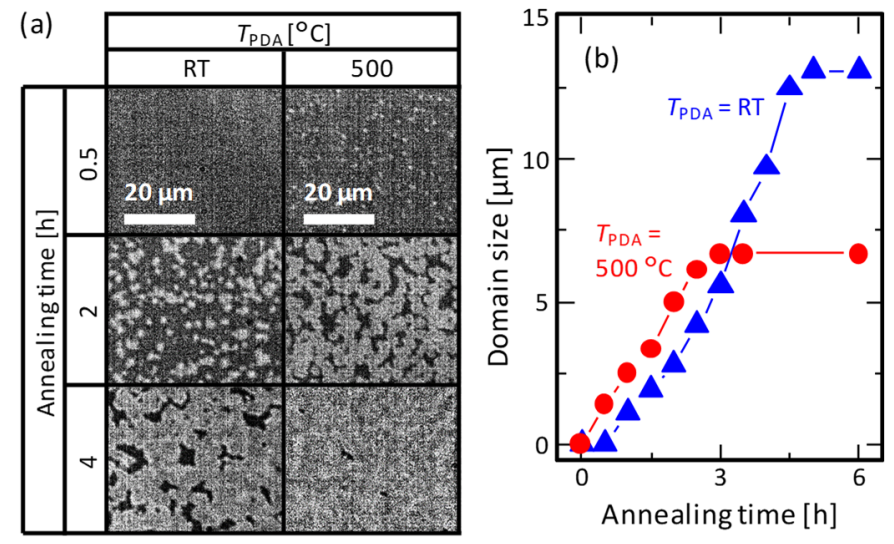

Fig. 2. Effects of PDA on the rate of SPC. (a) In-situ optical microscopy images of the samples annealed at $400{ }^{\circ} \mathrm{C}$. The dark-colored area indicates a-Ge, and the bright-colored area indicates c-Ge. (b) Annealing time dependence of the domain size derived from the micrographs. 

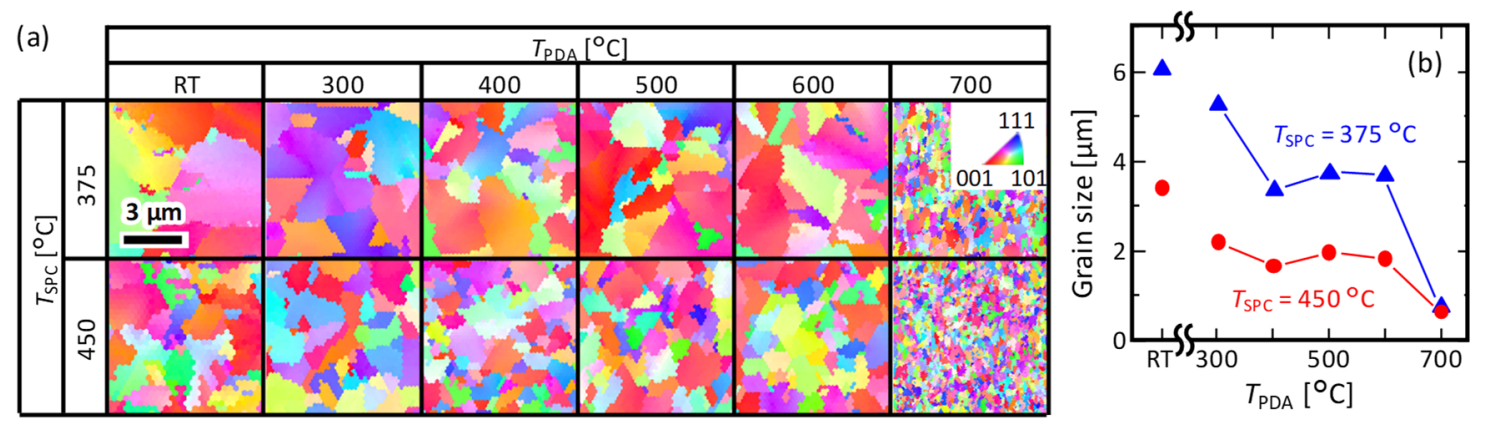

FIG. 3. (a) IPF images of the samples summarized as the matrix composed of $T_{\mathrm{SPC}}$ and $T_{\mathrm{PDA}}$. Colors indicate the crystal orientation, according to the inserted color key. (b) Average grain size determined by the EBSD analyses as a function of $T$ PDA. 

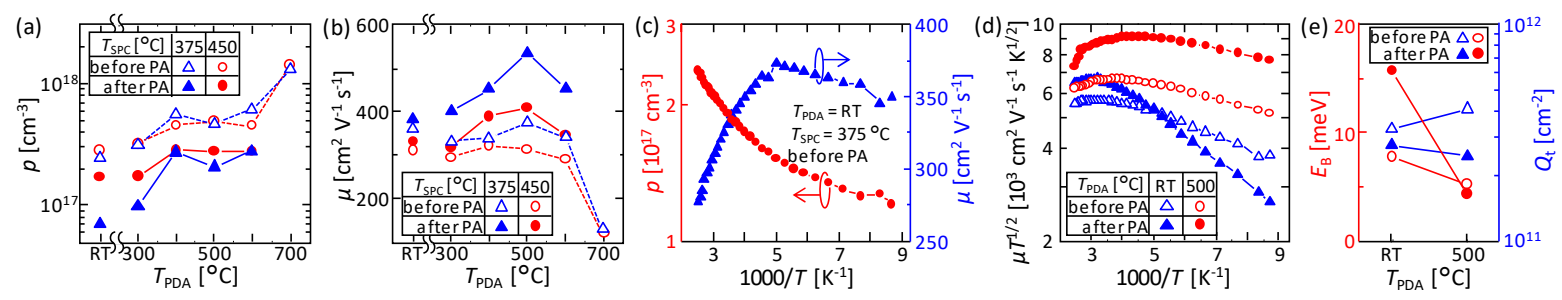

FIG. 4. Electrical properties of the SPC-Ge layers before and after PA. TPDA dependence of (a) hole concentration $p$ and (b) hole mobility $\mu$ for the samples with $T_{\mathrm{SPC}}=375$ and $475{ }^{\circ} \mathrm{C}$. (c) Arrhenius plot of $p$ and $\mu$ for the sample with $T_{\mathrm{PDA}}=\mathrm{RT}$ and $T_{\mathrm{SPC}}=375{ }^{\circ} \mathrm{C}$ before PA. (d) Arrhenius plot of $\mu T^{1 / 2}$ and (e) energy barrier height $E_{\mathrm{B}}$ and trap-state density $Q_{\mathrm{t}}$ for grain boundaries of the samples with $T_{\mathrm{PDA}}=\mathrm{RT}$ and $500{ }^{\circ} \mathrm{C}$, where $T_{\mathrm{SPC}}=375{ }^{\circ} \mathrm{C}$. 